\title{
XIII.
}

Aus der 1. inneren Abteilung des Städtischen Krankenhauses Charlottenburg-Westend.

(Prof. Umber.)

\section{Experimentelle Studien über die Beziehnng der urämischen Azotämie zur Indikanämie und Indikanurie.}

Von

\section{Dr. Max Rosenberg,}

Assistenten der Abteilung.

Die Indikanämie ist von 0 bermeyer und Popper ${ }^{1}$ ) entdeckt und in ihrem Verhältnis zur Urämie gewürdigt worden. Bei einer allerdings nur geringen Anzahl von Fällen konnte Dorner2) die Ergebnisse dieser Autoren bestätigen, Tschertk of ${ }^{3}$ ) hat dann aus unserer Abteilung an einem weit größeren Material das Verbältnjs der Azotämie zur Indikanämie und insbesondere anch deren prognostische Bedeutung klargestellt und durch Vereinfachung der Methodik die Anwendung der Indikanbestimmung im Serum für klinische Zweeke wesentlich erleichtert. Ich habe in Gemeinschaft mit Machwitz die von Tsehertk off begonnenen Untersuchungen fortgefthrt, und wir verfügen jetzt über das fünffache Material, das der Tschertkoffschen Arbeit zugrunde liegt. Wir werden unsere klinischen Resultate, die die Tschertk offsehen Angaben im Wesentlichen bestätigen und in mancher Hinsicht noch erweitern, demnächst an anderer Stelle veröffentlichen, ich möchte hier nur an der Hand von Tierexperimenten einen Beitrag zu der Frage liefern, welches die Ursache der Indikanämie ist und in welchen ätiologischen Beziehungen sie zur Azotämie steht.

1) Zeitschr. f. klin. Medizin Bd. 72, s. 371 .

2) D. Arch. f. klin. Med. 1914.

3) D. med. Wochenschr. 1914, Nr. 36. 
Wir haben uns von vornherein - wie wohl auch die anderen Autoren, die sich mit diesem Thema befaBten - bei dem Worte Indikanämie nicht das Vorhandensein von Indikan im Blut, sondern eine Erhöbung des Blutindikans vorgestellt, ebenso wie man unter Azotämie nicht das Vorhandensein von Reststickstoff im Blut, sondern eine Erhöhung des Rest-N-Spiegels versteht. Die Indikanämie sensu strictiori ist ebenso physiologiseh wie die Indikanurie; da das Blut ja das Transportmittel des Indikans von seiner Bildungsstätte zu seiner Exkretionsstätte darstellt. Wir speziell haben von Indikanämie dann gesprochen, wenn der Indikanspiegel im Blut diejenige Höhe erreichte, die sich mit der Obermeyer-Tschertkoff schen Methode nachweisen läßt.

Mit der verfeinerten Indikanbestimmung von Jolles ${ }^{1}$ ) gelingt es nun, wie auch schon $\mathrm{Haas}^{2}$ ) erwähnt, in jedem Blut Indikan nachzuweisen, und es entsteht die Frage, von welcher Höhe ab man den Indikanspiegel des Blutes als pathologisch bezeichnen soll, insbesondere aber, von welcher Höhe ab er als pathognomonisch für eine bestehende Azotämie anzusehen ist. Auf diese Frage werden wir an anderem Ort zuriickkommen, hier sei nur darauf hingewiesen, daß sich auch bei Nierengesunden eine Erhöhung des Indikanspiegels im Blut mit der Jollesschen Methode bei vermehrter Indikanbildung

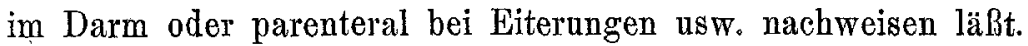

Die Entstehung dieser »Hyperindikanämie — ich benutze von jetzt ab zur Vermeidung von Mißverständnissen dieses Wort in demselben Sinne wie bisher Indikanämie $=$ Erhöhung des Indikanspiegels im Blut über den normalen Wert - bereitet dem Verständnis weiter keine Schwierigkeiten; sie beruht auf der Resorption des mehr gebildeten Indikans. Weit schwieriger gestaltet sich die Erklärung der Hyperindikanämie bei Azotämikern. Theoretisch läßt sich eine Hyperindikanämie auf vermehrte Bildung oder auf behinderte Ausscheidung zurückführen und auf den ersten Blick möchte es scheinen, als ob die zweite Ursache die richtige Erklärung für die Hyperindikanämie der Nephritiker gäbe. Aber schon bei den perakuten Fällen ron Urämie, die unter Anurie in $2-4$ mal 24 Stunden zum Tode führen (z. B. Sublimatvergiftungen), muB man zweifeln, ob diese Anschauung zutrifft, denn die nach 24 Stunden im Blute nachweisbare Indikanmenge ist oft so gro B (z. B. Sublimatkaninchen 3), daß sie unmöglich durch verhinderte Ausscheidung der

1) Ztschr. f. physiol. Chemie Bd. 94 und 95.

2) Münchn. med. Wochenschr. 1915, Nr. 31. 
geringen Indikanmengen entstanden sein kann, die sich im Harn, und besonders im Kaninchenharn, normalerweise finden. Die weiteren Ausführungen sollen zeigen, daß die Indikanretention nur in einem Teil der Fälle und in einem bestimmten Stadium die Hauptursache für das Zustandekommen der Hyperindikanämie bei der akuten Urämie bildet.

Für das Studium der Hyperindikanurie und Hyperindikanämie ist der Mensch ein schlechtes Versuchsobjekt, da wir die Menge des im Darm physiologisch gebildeten und resorbierten Indikans schwer beurteilen und kaum auf gleichem Niveau halten können. Nach den Untersuchungen von $\left.\operatorname{Rosin}^{1}\right)$ und von Harnack ${ }^{2}$ haben normal genährite gesunde Kaninchen einen indikanfreien Harn. Salkowski ${ }^{3}$ ) hingegen konnte bei Verwendung einer verfeinerten Methodik durch Anreicherung auch im normalen Kaninchenharn Spuren von Indikan nachweisen, doch waren diese so gering, daß man praktisch von einem indikanfreien Harn sprechen kann. Ich habe bëi meinen Versuchen an etwa 30 Kaninchen bei gesunden Tieren nie eine Indikanurie bei Anwendung der Obermeyerschen Probe finden können, mit Ausnahme eines einzigen Tieres, das spontan eine starke Indikanurie zeigte, ohne daß sich ein Grund dafür nachweisen ließ. Da zu dieser Zeit eine Seuche in unserem Kaninchenstall herrschte, bin ich geneigt, in diesem Fall die Indikanurie auf eine in vivo nicht nachweisbare Erkrankung zurïckzufuihren. Mit der Jollesschen Indikanprobe - ich habe die Thymolprobe im allgemeinen der $a$-Naphtholprobe vorgezogen - gaben die Kaninchenurine teils negative, teils zweifelhafte, bisweilen auch schwach positive Reaktionen. Ich habe für die Frage der Indikanurie stets die Obermeyersche Reaktion benutzt unter Verwendung von $10 \mathrm{ccm}$ Urin, $10 \mathrm{ccm}$ Obermeyers Reagens und $2 \mathrm{ccm}$ Chloroform. Fiel diese Reaktion zweifelhaft aus, so wiederholte ich sie mit $50 \mathrm{ccm}$ Urin, $50 \mathrm{ccm}$ Obermeyers Reagens und 3-4 ccm Chloroform. War nur die letztere Probe schwach positiv, so wurde die Reaktion als Spur bezeichnet, für stärkere Grade wandte ich die Bezeichnungen: sehwach,++ und ++ an. Die Jollessche Probe habe ich als für diese Zwecke zu fein gefunden. Wenn ich also im Folgenden von fehlender Indikanurie spreche, ist damit gemeint, daß die Indikanreaktion im Harn bei Anwendung der eben besprochenen Proben negativ war.

1). Virehows Archiv Bd. 123, 1891.

2) Ztschr. f. physiol. Chemie Bd. 29, 1900.

3) Ebenda Bd. 57, 1908. 
Die Indikanbestimmung im Serum wurde nach der ObermeyerTschertk offschen Mikromethode mit $2 \mathrm{ccm}$ Serum-Trichloressigsäurefiltrat, $2 \mathrm{ccm} O \mathrm{~b}$ ermeyers Reagens und $0,5 \mathrm{~cm}$ Chloroform in schmalen kleinen Reagensgläschen ausgeführt. Die Bezeichnungen Blutindikan $\emptyset,+$ und ++ sollen also in den folgenden Versuchen nur bedeuten, daß diese Indikanprobe negativ, positiv oder stark positiv war und, wie die Indikanproben im Urin, nur einen quantitativen, keinen qualitativen Unterschied zum Ausdruck bringen. Der Blutharnstoff wurde nach Ambard and Hallion bestimmt, so wie wir die Bestimmung auch beim Menschen ausführen (Näheres s. Machwitz und Rosenberg, D. Med. Woch. 1915, Nr. 38, S. 1123).

Die Untersuchungen hätten vielleicht noch klarere Resultate ergeben, wenn der Blutharnstoff der Kaninchen noch häufiger untersucht worden wäre, als dies bei unseren Tieren geschah. Es war jedoch leider nicht möglich, eine hänfigere Blutentnahme vorzunehmen, um die Tiere dadurch nicht zu sehr zu schwächen oder eine zu große Abnahme der Gesantblutmenge zu bewirken. Durch verschiedene Kontrollbestimmungen an gesunden Kaninchen üherzengten wir uns, daß der Blutharnstoff bei diesen Tieren etwa in denselben Grenzen wie beim Menschen schwankt; wir fanden Werte zwischen 0,20 und 0,60 pro mille.

Das Futter der Tiere bestand im wesentlichen aus Kraut und Rüben. Eine absolut gleichbleibende, quantitativ gemessene Nahrung ließ sich wegen der Knappheit an Viehfutter and Personal leider nicht zuführen. Der Urin wurde in 24 stündigen Portionen auf Eiweiß und Indikan untersucht; außerdem wurden tägliche $\mathrm{N}$-Bestimmungen nach Kjeldahl und tägliche NaCl-Bestimmungen nach Volhard vorgenommen. Um in eiweißhaltigem Urin eine Indikanbildung durch Fäulnis zu verhindern, wurde ein Thymolkrystall in die Uringläser geworfen.

Wenn man nun bei Kaninchen eine experimentelle Nephritis setzt, die zur Azotämie ${ }^{1}$ ) führt, so sieht man, daß bei Erhöhung des Harnstoffspiegels im Blut von etwa 1 pro mille an eine Indikanurie auftritt. Ich habe in gemeinschaftlicher Arbeit mit cand. med. P. Beeck experimentelle Vergiftungen bei Kaninchen studiert, die durch Oxalsäure, Uran, Sublimat, Chrom und Cantharidin hervorgerufen waren. Wir behalten uns die ausführliche Besprechung dieser Versuche an anderer Stelle vor, hier möchte ich sie nor soweit erwähnen, als sie zur Indikanfrage Beziehung haben.

1) Ich gebrauche im folgenden die Ausdrüicke Azotämie und Urämie, oder besser echte Urämie, als Synonyma und bezeichne damit jede renal bedingte Erhöhung des Blutharnstoffs iiber die Norm. Eine Wesensverschiedenheit zwischen der Azotämie durch Vergiftungsnephrosen nud bei akuten Glomerulonephritiden besteht unserer Ansicht nach nicht. (Näheres hieriber siehe meine oben erwähnte, demnächst erscheinende gemeinsame Arbeit mit $\mathrm{Machwitz}$ ) 
Experimentelle Studien über die Beziehung der urämischen Azotämie usw. 269

Ich gebe zunächst einen Auszug aus den Versuchsprotokollen:

\section{Oxalsänre.}

1. $3467 \mathrm{~g}$ schweres Kaninchen erhält 0,12 Natriumoxalat intravenös. In den folgenden Tagen im Urin Spuren Eiweiß, kein Indikan. Blatharnstoff bleibt normal. Keine Krankheitserscheinungen.

2. $2860 \mathrm{~g}$ schweres Kaninchen erhält 0,15 Natriumoxalat intravenös. Nach 24 Stunden im Urin Spuren Eiweiß, kein Indikan. Blutharnstoff 0,98 pro mille, kein Indikan im Blut. Am nächsten Tag im Urin Spuren Eiweiß, Indikan + . Am dritten Tage ist Eiweiß und Indikan geschwunden, Tier gesund.

3. $3250 \mathrm{~g}$ schweres Kaninchen erhält 0,15 Natriumoxalat intravenös:

\begin{tabular}{|c|c|c|c|c|c|}
\hline \multirow{2}{*}{ Tag } & \multirow{2}{*}{$\begin{array}{c}\text { Natriumoxalat- } \\
\text { Zufuhr }\end{array}$} & \multicolumn{2}{|c|}{ Urin } & \multicolumn{2}{|l|}{ Blut } \\
\hline & & Albumen & Indikan & Harnstoff $\%$ & Indikan \\
\hline 1. & 0,15 intravenös & + & $\emptyset$ & - & - \\
\hline 2. & - & Spur & $\emptyset$ & 24 Std. post inj. 0,2 & $\not$ \\
\hline 3. & 0,15 intravenös & schwach + & + & - & - \\
\hline 4. & - & schwach + & $+t$ & - & - \\
\hline 5. & - & Spur & sehwach + & 48 Std. post inj. 1,29 & $\emptyset$ \\
\hline 6. & - & $\emptyset$ & $\emptyset$ & - & - \\
\hline 7. & 0,12 intravenös & + & $\emptyset$ & 一 & - \\
\hline 8. & - & sehwach + & $\emptyset$ & - & - \\
\hline 9. & - & $\emptyset$ & $\emptyset$ & 48 Std. post inj. 0,50 & $\emptyset$ \\
\hline
\end{tabular}

4. $2477 \mathrm{~g}$ schweres Kaninchen erhält 0,165 Natriumoxalat intravenös. Am ersten Tage anurisch, Blutharnstoff nach 24 Stunden 1,91 pro mille, kein Indikan im Blut nachweisbar. Am zweiten Tage im Urin Eiweiß schwach + , Indikan Spur. Nach 48 Stunden zweite Injektion von 0,18 Natriumoxalat, dabei Exitus. Blutharnstoff unmittelbar post mortem 2,42 pro Mille, Blutindikan + .

5. $3100 \mathrm{~g}$ schweres Kaninchen erhält 0.18 Natriumoxalat intravẹnös:

\begin{tabular}{|c|c|c|c|c|c|}
\hline \multirow{2}{*}{ Tag } & \multirow{2}{*}{$\begin{array}{c}\text { Natriumoxalat- } \\
\text { Zufuhr }\end{array}$} & \multicolumn{2}{|c|}{ Urin } & \multicolumn{2}{|l|}{ Blut } \\
\hline & & Albumen & Indikan & Harnstoff $\%$ & Indikan \\
\hline 1. & 0,18 intravenös & Spur? & $\emptyset$ & - & - \\
\hline 2. & - & $\emptyset$ & $\emptyset$ & 24 Std. post inj. 1,13 & $\emptyset$ \\
\hline 3. & 0,105 intravenös & Spur & $\emptyset$ & - & - \\
\hline 4. & - & $\emptyset$ & $\varnothing$ & $\ldots$ & - \\
\hline 5. & - & $\emptyset$ & $g$ & - & - \\
\hline 6. & 0,135 intrayenös & Spur & 9 & - & - \\
\hline 7. & - & $\emptyset$ & $\theta$ & 24 Std. post inj. 0,20 & $\emptyset$ \\
\hline 8. & 0,3 subkutan & $\emptyset$ & $\emptyset$ & - & - \\
\hline 9. & - & $\not$ & $\emptyset$ & 一 & - \\
\hline
\end{tabular}


Es ist dies der einzige Fall, wo bei erhöhtem (1,13 pro mille), aber schnell absinkendem Blutharnstoff keine Indikanurie auftrat. Er gehörte zu unseren ersten Versuchen und wir führten damals nur die Obermeyersche Probe mit $10 \mathrm{~cm}$ Urin aus; vielleicht hätte die Probe mit $50 \mathrm{ccm}$ Urin ein positives Resultat ergeben.

\section{Uran.}

1. $1920 \mathrm{~g}$ schweres Kaninchen erhält 0,016 Uranylazetat subkutan:

Urin

Tag

1.

2.

3.

4.

Blut

Albumen Indikan Harnstoff $\%$ Indikan

5. Exitus. In der Blase wenige Kubikzentimeter Urin: Albumen ++ , Indikan schwach + . Im Blut sofort post mortem 6,38 pro mille Harnstoff, Indikan ++ .

2. $2025 \mathrm{~g}$ schweres Kaninchen, dessen Urin bei dreitägiger Beobachtung indikanfrei war, erhält 0,01 Uranylazetat subkutan:

\section{Urin}

Tag

1.

2.

3.

4.

5 .

6.

7.

$\begin{array}{cc}\text { Menge Albumen } & \text { Indika } \\ \varnothing & \emptyset \\ \varnothing & \emptyset \\ 6 \% & + \\ 16 \% & \varnothing \\ 131 / 2 \% & \varnothing \\ 4 \% \% & \varnothing \\ \text { schwach }+ & \varnothing\end{array}$

)

0

$\theta$

$\theta$

$\emptyset$
Blat

Harnstoff $\%$ Indikan

2,57 schwach +

$5,85 \quad+$

$9,00 \quad++$ post mortem.

Wir sehen also hier bei rasch ansteigender Azotämie eine Indikanurie auftreten, die aber schnell wieder verschwindet. Wir glauben diese Erscheinung bei Berücksichtigung der weiter unten za besprechenden Fälle so denten zп müssen, daß die Nierenfunktion, wie auch der rapid ansteigende Blutharnstoff und die schnell sinkenden Urinmengen zeigen, so gelitten hat, daß die Indikanpassage vollkommen gesperrt ist. Die Hyperindikanämie als Folge von Indikanstauung im Blut kommt also bei der akuten Azotämie vor, die Staung spielt aber nur bei den schwersten Graden der Niereninsuffizienz die Hauptrolle.

3. $2345 \mathrm{~g}$ schweres Kaninchen, dessen Urin bei zweitägiger Beobachtung indikanfrei war, erhält 0,004 Uranylazetat subkutan: 
Experimentelle Studien über die Beziehung der urämischen Azotämie usw. 271

Urin

Tag

1.

2.

3. schwach +

4.

5.

6.

7.

8.

9.

10.

$\begin{array}{cc}\text { Albumen } & \text { Indikan } \\ \emptyset & \emptyset \\ \text { schwach }+ & \emptyset \\ \text { schwach }+ & + \\ \text { schwach }+ & + \\ 1 / 2 \% 00 & ++ \\ \text { schwach }+ & ++ \\ \text { Spur } & + \\ \emptyset & \emptyset \\ \emptyset & \emptyset \\ \emptyset & \emptyset\end{array}$

0

3,05

$\emptyset$

0,45

0

Einige Tage darauf erhält das von der ersten Injektion geheilte Tier 0,006 Uranylazetat subkutan:

\begin{tabular}{|c|c|c|c|c|}
\hline \multirow[b]{2}{*}{ Tag } & \multicolumn{2}{|c|}{ Urin } & \multicolumn{2}{|c|}{ Blut } \\
\hline & Albumen & Indikan & Harnstoff $\%$ & Indikan \\
\hline 1. & Spur & 0 & & \\
\hline 2. & $1 \%$ & 0 & & \\
\hline 3. & $13 / 4 \%$ & $\varnothing$ & & \\
\hline 4. & $1 / 2 \%$ & $\theta$ & & 0 \\
\hline 5. & Spur & Spur & 0,97 & ט \\
\hline 6. & Spur & Spur & & \\
\hline 7. & Spur? & Spur & & \\
\hline 8. & Spur & Spur? & & \\
\hline 9. & Spur & $\theta$ & & \\
\hline 10. & Spur & Spur & & \\
\hline 11. & 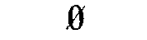 & $\emptyset$ & 0,58 & $\theta$ \\
\hline
\end{tabular}

Am nächsten Tage erhält das Tier 0,01 Uranylazetat subkutan. Darauf tritt eine Spur Eiweiß im Urin auf, der Blutharnstoff bleibt normal $(0,20$ pro mille), keine Indikanurie. Einige Tage darauf erbält das Tier nach Abklingen der Albuminurie die gleiche Dosis einer frischen Lösung. Darauf erfolgt wieder eine in 3 Tagen abklingende, am ersten Tag 1 pro mille erreichende Albuminurie, keine Indikanurie, der Blutharnstoff beträgt am zweiten Tage nach der Vergiftung 0,50 pro mille. Die Injektion von 0,02 Uranylazetat, die 10 Tage später erfolgt, bewirkt nur eine 6 . Tage anhaltende quantitativ nicht meßbare Albuminurie, keine Indikanurie; Blntharnstoff nicht bestimmt, aber wahrscheinlich normal. Nach Abklingen der Albuminurie erhält das Tier 0,04 Uranylazetat subkutan: 


\begin{tabular}{|c|c|c|c|c|}
\hline & \multicolumn{2}{|c|}{ 1] rin } & \multicolumn{2}{|c|}{ Blut } \\
\hline Tag & Albumen & Indikan & Harnstoff $\%$ & Indikan \\
\hline 1. & $11 / 3 \%$ & Spur & & \\
\hline $\begin{array}{l}2 . \\
3 . \\
4 .\end{array}$ & $\begin{array}{l}1 \% \\
3 / 4 \% \\
\text { Snur }\end{array}$ & $\begin{array}{l}+ \\
+ \\
\text { Snur? }\end{array}$ & 2,60 & + \\
\hline 5. & $\emptyset$ & Spur & 310 & + \\
\hline 6. & $\theta$ & + & 0.10 & + \\
\hline $\begin{array}{l}7 . \\
8 .\end{array}$ & $\begin{array}{l}\text { Spur? } \\
\text { Spur }\end{array}$ & $\begin{array}{c}\text { schwach } \\
\text { Spur }\end{array}$ & 6,25 & ++ \\
\hline
\end{tabular}

Die Indikanurie tritt also auch hier erst bei Einsetzen der Azotämie auf, um bei zunehmender Niereninsuffizienz (steigender Azotämie) zurückzugehen. Das Retentionsmoment kommt also in diesem letzten Stadium als wesentlicher Faktor für die Entstehung der Hyperindikanämie zu der vermehrten Indikanbildung hinzn.

Sublimat.

1. Etwa $2500 \mathrm{~g}$ schweres Kaninchen erhält 0,008 Sublimat intravenös:

Urin

\begin{tabular}{|c|c|c|}
\hline Tag & Albumen & IndikaI \\
\hline 1. & $31 / 2 \%$ & $\gamma$ \\
\hline 2. & $3 \%$ & $\emptyset$ \\
\hline 3. & $11 / 2 \%$ & + \\
\hline $\begin{array}{l}4 . \\
5 .\end{array}$ & $\begin{array}{r}1 / 4 \% 00 \\
\text { an }\end{array}$ & $\mathrm{sech}^{+}$ \\
\hline 6 . & Spur & + \\
\hline 7. & Spur & Spur \\
\hline 8. & Spur & Spur? \\
\hline 9. & \multicolumn{2}{|c|}{ kein Urin } \\
\hline 10. & Spur & $\emptyset$ \\
\hline
\end{tabular}

Blut

Harnstoff $\%$ Indikan

2,22

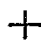

2,84

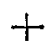

$\emptyset$

Das gleiche Tier erhält darauf 0,006 Sublimat intravenös: Am ersten Tage anurisch. Blutharustoff nach 24 Stunden 0,98 pro mille, Blutindikan $\varnothing$, am zweiten Tage enthält der Urin Spuren Eiweiß und Spuren Indikan, am dritten und vierten ebenso. Am fünften und sechsten Tage immer noch Spuren Eiweiß, aber kein Indikan im Urin, am Abschluß des sechsten Tages findet sich im Blut 0,30 pro mille Harnstoff, kein Indikan. Heilung.

2. $2705 \mathrm{~g}$ schweres Kaninchen erhält 0,01 Sublimat intravenös. Es ist darauf 3 Tage lang anurisch und stirbt. Der Blutharnstoff beträgt kurz ante mortem 5,55 pro mille, Indikan im Blut ++ . Post mortem fand sich in der Blase etwa $1 / 4$ Urin, in demselben eine nicht sicher positive Indikanreaktion (Spur?). Hier war also die Niereninsuffizienz so groß, daß von vornherein neben der vermehrten Indikanbildung die Indikanretention eine Hyperindikanämie verursachte. 
3. $2350 \mathrm{~g}$ schweres Kaninchen erhält 0,08 Sublimat per os mit der Magensonde. Nach 1 Stunde läßt es $20 \mathrm{ccm}$ Harn, der frei von Indikan ist und Spuren Eiweiß enthält (offenbar im ersten Beginn der Nierenwirkung des Sublimats sezerniert), ist dann anurisch. Nach 24 Stunden beträgt der Blutharnstoff 2,7 pro mille, nach 48 Stunden 5,06 pro mille, beim Exitus, der nach 72 Stunden eintritt, 6,6 pro mille. Die Indikanämie ist schon nach 24 Stunden ++ und steigt ständig; so daß beim Tode die Reaktion nach Obermeyer noch bei 10 facher Verdünnung des Filtrats positiv ist. Es ist dies der einzige Fall, in dem wir schwere blutige Durchfälle verzeichnen konnten. Hier war nun die Hyperindikanämie so kolossal, daß vielleicht auch an eine vermehrte Indikanbildung im Darm bei gehemmter Ausfuhr gedacht werden kann.

Die weiteren Versuche mit Sublimatvergiftungen sind für die Indikanfrage nicht branchbar, weil das Sublimat subkutan injiziert worde. Es entstanden dabei Infiltrate und Abszesse, die ihrerseits zur Bildung von Indikan führten.

\section{Chrom.}

1. $2059 \mathrm{~g}$ schweres Kaninchen erhält, nachdem der Urin während 24 stündiger Beobachtung frei von Eiweiß und Indikan gefunden wurde, 0,08 Chromkali subkutan. Keine Abszeßbildung:

\begin{tabular}{ccccc} 
& \multicolumn{2}{c}{ Urin } & \multicolumn{2}{c}{ Blut } \\
Tag & Albumen & Indikan & Harnstoff $\%$ & Indikan \\
$\mathbf{1 .}$ & $11 / \mathbf{4} \%$ & ++ & & \\
$\mathbf{2}$ & $\mathbf{5} \% 00$ & ++ & 1,10 & $\emptyset$ \\
3. & anurisch & & 4,50 & + \\
4. & anurisch & & &
\end{tabular}

Am Schluß des vierten Tages Exitus. Blutharnstoff 6,90 pro mille, Blutindikan ++ .

$$
\text { Cantharidin. }
$$

1. $2228 \mathrm{~g}$ schweres Kaninchen erhält 0,005 Cantharidin (1\% Lösung in Essigäther) subkutan, keine Abszeßbildung:

Urin

Blut

$\begin{array}{ccccc}\text { Tag } & \text { Albumen } & \text { Indikan } & \text { Harnstoff } \% & \text { Indikan } \\ 1 . & \emptyset & \emptyset & & \\ 2 . & \text { Spur } & ++ & 0,80 & \emptyset \\ 3 . & \text { Spur } & ++ & & \\ 4 . & \emptyset & ++ & 0,69 & \emptyset \\ 5 . & \emptyset & ++ & & \\ 6 . & \emptyset & + & 0,25 & \emptyset \\ 7 . & \emptyset & \emptyset & & \\ 8 . & \emptyset & \emptyset & & \end{array}$


2. $1735 \mathrm{~g}$ schweres Kaninchen erhält, nachdem der Urin 3 Tage lang indikanfrei gefunden wurde, 0,004 Cantharidin subkutan, keine Abszeßbildung:

\begin{tabular}{ccccc} 
& \multicolumn{2}{c}{ Urin } & \multicolumn{2}{c}{ Blut } \\
Tag & Albumen & Indikan & Harnstoff $\%$ & Indikan \\
1. & schwach + & Spur? & & \\
2. & $\varnothing$ & $\varnothing$ & 0,20 & $\emptyset$ \\
3. & $\varnothing$ & $\emptyset$ &
\end{tabular}

Am vierten Tage erhält das Tier 0,006 Cantharidin subkutan. Am nächsten Morgen wird es tot aufgefunden. Im Urin fragliche Spuren von Eiweiß, kein Indikan. Im Blat post mortem 0,40 pro mille Harnstoff, kein Indikan.

3. $1995 \mathrm{~g}$ schweres Kaninchen erhält, nachdem der Urin während 24 Stunden frei von Indikan gefunden wurde, 0,005 Cantharidin subkutan. Keine Abszeßbildung:

\begin{tabular}{|c|c|c|c|c|}
\hline \multirow[b]{2}{*}{ Tag } & \multicolumn{2}{|c|}{ Urin } & \multicolumn{2}{|c|}{ Blut } \\
\hline & Albumen & Indikan & Harnstoff $\%$ & Indikan \\
\hline 1. & $11 / 2 \%$ & 0 & & \\
\hline 2. & $\begin{array}{l}1 / 2 \% \\
10 \%\end{array}$ & $\underline{0}$ & & \\
\hline $\begin{array}{l}3 . \\
4 .\end{array}$ & $\begin{array}{l}1 \% 00 \\
3 \% 00\end{array}$ & 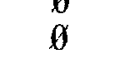 & 0 & $\theta$ \\
\hline 5. & Spur & $\emptyset$ & 0,00 & 0 \\
\hline 6. & D & $\theta$ & & \\
\hline
\end{tabular}

Ans den eben mitgeteilten Versuchen geht unseres Erachtens deutlich hervor, daß bei mit Oxalsäure, Uran, Sublimat, Chrom und Cantharidin vergifteten Kaninchen eine Indikanurie auftritt, die vorher nicht vorhanden war, bzw. daß eine ganz geringe, mit der Obermeyerschen Probe nicht nachweisbare Indikanurie so stark ansteigt, daß sie mit dieser Probe nachweisbar wird. Dieses Ansteigen der Indikanurie tritt fast ausnahmslos auf, sobald der Blutharnstoff auf 1 pro mille steigt, zuweilen etwas früher, zuweilen etwas später. Der Punkt, bei dem die vermehrte Indikanurie einsetzt, läßt sich bei der zuweilen sehr steil ansteigenden Blutharnstoffkurve nicht immer genau bestimmen, zumal da der wiederholten Blutentnahme beim Kaninchen wegen der geringen Gesamtblutmenge des Tieres natürliche Grenzen gesetzt sind. Jedenfalls tritt aber gleichzeitig mit der Erhöhung des Blutharnstoffes eine vermehrte Indikanbildung auf. Dabei kommt es auch naturgemäß, wie bei jeder Hyperindikanurie, zu einer Erhöhung des Blutindikans, doch ist diese zunächst nicht so beträchtlich, daß sie sich mit der Obermeyer-Tschertk offschen Mikromethode, wohl aber 
mit der Jollesschen Probe, nachweisen läBt. Erst bei weiter zunehmender Niereninsuffizienz steigt dann das Blutindikan zu höheren Werten und schließlich bei schwerer Insuffizienz, besonders natürlich bei völliger Anurie, versiegt die Indikanurie völlig, so daß sich dann zu der vermehrten Indikanbildung eine dauernd zunehmende Indikanretention gesellt. Wir haben es also bei der akuten Urämie der Kaninchen infolge der oben beschriebenen Nierenschädigung zunächst mit einer Mehrbildung von Indikan zu tun, die die Hyperindikanurie und Hyperindikanämie zur Folge hat. Erst später gesellt sich zu der vermehrten Indikanbildung eine Indikanretention, so daß die Verknüpfung beider Ursachen ein weiteres Ansteigen der Hyperindikanämie zur Folge hat.

Es drängt sich nun natürlich die Frage auf, woher das mehrgebildete Indikan stammt. Die Bildung von Indikan im Organismus kann - darïber sind alle Autoren einig -- entstehen: 1, enteral durch Fäulnis des per os zugeführten oder aus den Verdaungsdrüsen in den Darm ergossenen Eiweißes, 2. parenteral durch bakteriellen Zerfall von Eiweiß bei Gangrän, Abszessen, jauchigen Eiterungen, putrider Bronchitis usw. Ob eine Indikanbildung auch durch abakteriellen parenteralen Eiweilzerfall zustande kommen kann, ist noch nicht sichergestellt. Schon Jaffé $\left.{ }^{1}\right\rangle$, dem wir die ersten grundlegenden Untersuchungen ïber. Indikanbildung und -ausscheidung: verdanken, hat diese Frage erörtert, und die von Senator beobachtete starke Indikanurie bei Karzinomkranken ist vielfach im Sinne einer Indikanbildung aus abakteriell zerfallenem Körpereiweiß gedeutet worden. Harnack und von der Leyen, Moraczewski, Blumenthal, Rosenfeld und Lewin sind anf Grund ihrer Untersuchungen für diese Hypothese eingetreten, während sie von Scholz, P. Mayer, Ellinger und auch von Jaffé als nicht bewiesen angesehen wird. Besonders Magnus-Lery ${ }^{2}$ ) ist sehr energisch dagegen eingetreten, da es ihm nnwahrscheinlich erscheint, daB zerfallendes Körpereiweiß andere Spaltprodukte bilden soll, als es dies bei der Salzsäurespaltung, bei der Trypsinverdaung und der Autolyse tut.

In unserem Falle kommt eine bakterielle parenterale Indikanbildung nicht in Frage. Wir haben die subkutane Injektion besonders schwer löslicher und stark reizender Stoffe (Oxalsäure, Sublimat) möglichst vermieden, und wenn wir subkatane Injektionen Bd. II.

1) Literatur s. bei Neubauer-Huppert, Analyse des Harns 11. Aufl,

2) v. Noorden, Pathologie des Stoffwechsels Bd. I. 
machten, sie stets unter allen aseptischen Kautelen ausgeführt. Bildeten sich trotzdem, wie wir es anfangs bei subkutaner Zufuhr von Sublimat oder Oxalsäure beobachteten, Abszesse oder Infiltrate, so haben wir diese Tiere von vornherein aus unseren. Versuchen ausgeschaltet und sind dann bei diesen Giften zur intravenösen oder oralen Applikation übergegangen. Es liegt nun die Annahme nahe, daß eine vermehrte Indikanbildung im Darm die Quelle der Hyperindikanurie und Hyperindikanämie sèi, weil nämlich ein Teil der von uns benutzten Gifte (Quecksilber, Chrom, Uran) als Schwermetalle im Dickdarm ausgeschieden, hier eine Entziundung der Schleimhaut hervorrufen und anf diesem Wege eine vermehrte Indikanbildung bewirken können. Wir halten diese Annahme für möglich, besonders in vereinzelten Fällen, bei Verabfolgung großer Dosen der lokal reizenden Gifte per os (Sublimatkaninchen 3). Wollte man sie weiter ausfiuhren, so könnte man bei der nicht auf Vergiftung basierenden akuten Urämie des Menschen, für die die Verhältnisse nach unseren bisherigen Erfahrungen ganz analog liegen wie bei den experimentellen Kaninchenephritiden, an die urämische Colitis denken, die ja auch anatomisch der $\mathrm{Hg}$-Colitis sehr ähnelt. Wir haben zwar bei unserem nachgerade sehr reichlichen Urämiematerial die urämische Colitis klinisch und anatomisch recht selten beobachten können, aber es ließe sich immerhin denken, daß es für die vermehrte enterale Indikanbildung einer ausgesprochenen Colitis nicht bedürfe, sondern daß vielleicht klinisch und anatomisch weniger markante Vorstufen derselben genügten. Eine Entscheidung dieser Frage läßt sich unseres Erachtens zur Zeit noch nicht treffen, aber immerhin hat diese Erklärung der vermehrten Indikanbildung für uns doch etwas Unbefriedigendes. Denn erstens findet sich die Indikanurie bei den, parenteral appliziert, den Darm wenig schädigenden Giften Kantharidin und Oxalsäure genan in gleicher Weise wie bei den zur Colitis führenden Giften Sublimat, Uran und Chrom, sobald eine Azotämie auftritt. Zweitens führen oft gleiche Dosen desselben Giftes bisweilen zur Indikanurie, bisweilen nicht, und zwar tritt die Indikanurie eben nur dann auf, wenn eine Azotämie entsteht ${ }^{1}$ ). Ferner haben wir intra vitam einen durchfälligen oder gar blutigen Stuhl bei den in Frage kommenden Tieren (mit Ausnahme des Sublimatkaninchen 3) nie beobachtet und auch post mortem eine nennenswerte entzündliche

1) Es ist doch anffallend, daß selbst bei wiederholter Sublimatinjektion (Sublimatkaninchen 2), wo sich also die Darmschädigungen häufen, es bei fehlender Azotämie nicht zur Indikanurie kommt, sondern diese eben immer nur auftritt, sobald der Blutharnstoff ansteigt. 
Veränderung des Dickdarms nicht feststellen können. Ferner ist hervorzuheben, daß als wesentlichste Quelle der Indikanbildung nicht der Dickdarm, sondern der Dünndarm in Frage kommt, und daß schließlich die angewandten Gifte meist stark bakterizid wirken und also durch Vernichtung der Darmflora die Indikanbildung eher herabsetzen als vermehren müßten. So gelang es auch Baumann ${ }^{1}$ ), bei Hunden durch große Kalomelgaben den Harn indikanfrei zu machen.

Um dieser Frage aber noch weiter nachzugehen, habe ich mehreren Kaninchen das Gift per os (Magensonde) zugeführt, weil auf diese Weise, wenn die reizende Substanz den ganzen Magen-Darmtraktus passiert, die Entzündung der Schleimhaut naturgemäß stärker sein muß, als bei subkataner oder intravenöser Applikation. Ich wählte dabei mittlere Dosen, um einerseits einen möglichst großen Schleimhautreiz zu setzen, andererseits das Auftreten einer Azotämie wie bei Sublimatkaninchen 3 zu vermeiden. Ich gebe diese Versuche im Folgenden wieder:

1. $1995 \mathrm{~g}$ schweres Kaninchen, dessen Harn indikanfrei war, erhält 0,1 Oxalsäure in Wasser gelöst per os. Es tritt keine Albuminurie auf, keine Indikanurie, keine Durchfälle. Das Tier bleibt gesund. Blutharnstoff 2 Tage nach der Vergiftung 0,2 pro mille.

2. $1900 \mathrm{~g}$ schweres Kaninchen, dessen Harn bei mehrtägiger Beobachtung indikanfrei war, erhält 0,04 Chromkali in reichlich Wasser gelöst per os. Es tritt eine geringe, schnell wieder vorübergehende Albuminurie auf, keine Indikanurie. Blutharnstoff bleibt normal. Keine Durchfälle. Das Tier bleibt gesund.

3. $3840 \mathrm{~g}$ schweres Kaninchen, dessen Urin bei zweitägiger Beobachtung indikanfrei war, erhält 0,04 Sublimat mit der Magensonde. Keine Albuminurie, keine Indikanurie. Blutharnstoff 2 Tage nach der Vergiftung 0,40 .

4. $1905 \mathrm{~g}$ schweres Kaninchen erhält 0,2 Oxalsäure in Wasser gelöst per os. Keine Albuminurie, keine Indikanurie, Blutharnstoff bleibt normal.

Trotz starker Darmreizung trat also bei diesen Tieren keine Hyperindikanurie anf, eben weil die Azotämie ausblieb. Es verliert damit also die Annahme, daß die Reizung der Darmschleimhaut die Ursache der Hyperindikanurie sei, viel an Wahrscheinlichkeit.

Auf Grund der vorstehenden Untersuchungen und Überlegungen scheint uns also die Annahme einer bakteriell enteral bedingten vermehrten Indikanbildung als Quelle fuir die Hyperindikanurie und Hyperindikanämie nicht befriedigend. Nun werde ich in einer dem-

1) Zeitschr. f. physiol. Chemie Bd.10, S. 123. 
nächst erscheinenden gemeinschaftlichen Arbeit mit Machwitz über den Stickstoffwechsel der Nephritiker beweisen können, daß bei der echten akuten Urämie (Azotämie), sowohl beim Menschen als auch in diesen Tierversuchen, ein vermehrter toxischer Eiweißzerfall aus den Stoffwechseluntersuchungen unbedingt angenommen werden muB, wie dies von Widal und seinen Schülern schon seit langem geschieht ${ }^{1}$ ). Bei Berücksichtigung dieser Tatsache erscheint nun die Annahme äußerst verlockend, daß der mehrgebildete Harnstoff und das mehrgebildete Indikan beide mit dem Zerfall des Körpereiweißes in Zusammenhang stehen, eine Anschaung, die bei unseren dürftigen Kenntnissen über die intermediären Stoffwechselprodukte im normalen und besonders im pathologischen Stoffwechsel zwar bisher nicht bewiesen ist, aber anch nicht mit Sicherheit widerlegt werden kann. Unsere Versuche über Mehrbildung von Indikan bei Eiweißzerfall infolge anderer Intoxikationen (Phosphor, Arsen) und Autointoxikationen (Coma diabeticum, maligne Tumoren) haben bisher noch zu keinem eindeutigen Resultat geführt. So trat bei mit Phosphor vergifteten Kaninchen einmal bei normalem Blutharnstoff eine vorïbergehende leichte Indikanurie auf, ein zweites Mal wurde sie vermißt. Ich möchte hinzufügen, daß die Verhältnisse der Indikanausscheidung bei der akuten Urämie des Menschen in den wenigen Fällen, die wir beobachtet haben, seitdem wir auch auf die Indikanurie genauer achten, ein den Tierversuchen durchaus paralleles Verhalten zeigen. Nur sind die Verhältnisse hier insofern verwickelter, als die physiologische Indikanurie beim Menschen stärker ist als beim Kaninchen und unsere quantitativen Indikanbestimmungen doch noch immer recht ungenau sind.

Wir glauben uns also zu der Annahme berechtigt, daß bei der akuten Urämie, sei es infolge akuter Glomerulonephritis, sei es infolge einer Nierenerkrankung durch Vergiftung, ein Zerfall von Körpereiweiß statt hat, bei dem es gleichzeitig zu einer vermehrten Indikanbildang vielleicht im Darm, wahrscheinlicher im intermediären Stoffwechsel

1) Diese Tatsache, daß bei der Urämie Körpereiweiß (Niereneiweiß?) zerfällt, hat vielleicht Berihhrungspunkte mit der alten Hypothese Ascolis von den Nephrolysinen, die ihrerseits wieder mit den neneren Vorstellungen Abderhaldens in Zusammenhang gebracht werden kann. Es findet sich iibrigens im Blute der Azotämiker neben der Hyperindikanämie auch eine Vermehrung anderer Eiweißspaltprodukte, woranf anch sehon Obermeyer nnd Popper aufmerksam gemacht haben. Ich fand besonders eine Vermehrung solcher abiureter Körper, die die Xanthoprotein-Reaktion, die Reaktion von Adamkiewicz and die Millon sche Reảktion geben. 
kommt. Es fragt sich nun, ob die Erhöhung des Rest-N- oder Harnstoffspiegels im Blut und die vermehrte Indikanbildung koordiniert sind, ob beide gleichzeitig anf denselben Reiz hin erfolgen, oder ob die eine Erscheinung durch die andere bewirkt wird.

Um dieser Frage näher zu kommen, haben wir bei nierengesunden Kaninchen eine vorübergehende künstliche Azotämie erzeugt, indem wir sie plötzlich mit großen Harnstoffdosen überlasteten. Wir führten anfangs den Harnstoff in $50 \%$ iger Lösung subkutan $\mathbf{z u}$, gingen aber bald zur stomachalen Applikation mit der Magensonde über, weil wir bei der Injektion dieser hochkonzentrierten Lösung zuweilen Infiltrate und Abszesse auftreten sahen, die ihrerseits für die Entstehnng einer etwa anftretenden Indikanurie in Frage kommen konnten. Wir gingen also so vor, daß wir 10 oder $20 \mathrm{~cm}$ (in einzelnen Fällen auch mehr) einer 50\% igen Harnstofflösung durch die Magensonde einführten. Es zeigte sich danach eine Erhöhung des Blutharnstoffs auf etwa 2 pro mille $(10 \mathrm{ecm})$ bzw. $3-4$ pro mille $(20 \mathrm{ccm})$, der sein Maximum nach etwa $2 \frac{1}{2}$ bis 6 Stunden erreichte und im Laufe ron 24 Stunden meist völlig zur Norm abgefallen war. Auch die N-Ansscheidungskurve im Urin bewies, daß über $90 \%$ des zugeführten $\mathrm{N}$ in 24 Stunden durch die Nieren ausgeschieden waren. Nur bei noch größeren Dosen und täglich wiederkehrender Überlastung des Tieres mit Harnstoff zeigte sich die Niere ibrer vermehrten Aufgabe nicht gewachsen (besonders bei Beschränkung der Flüssigkeitszufuhr), und das Tier ging an zanehmender Azotämie zugrunde, indem es Blutharnstoffwerte bis über 11 pro mille(!) zeigte. Nebenbei sei bemerkt, daß die Tiere bei Blutharnstoffwerten über 5 pro mille meist eine auffallend vertiefte und bis zur Hälfte des Normalen verlangsamte Atmung zeigten, die ganz der großen KuBmaulschen Atmung entsprach, die wir vom Coma diabeticum und uraemicum des Menschen kennen.

\section{Harnstoffrergiftungen.}

1. $3050 \mathrm{~g}$ schweres Kaninchen erhält $23 \mathrm{ccm}$ einer $50 \%$ igen UreaLösung subkutan:

\begin{tabular}{|c|c|c|c|c|c|}
\hline \multirow{2}{*}{ Tag } & \multirow{2}{*}{ Urea-Zufubr } & \multicolumn{2}{|c|}{ Urin } & \multicolumn{2}{|l|}{ Blut } \\
\hline & & Albumen & Indikan & Harnstoff $\% / 00$ & Indikan \\
\hline 1. & $11, \check{g} \mathrm{~g}$ subkutan & Spur & $\emptyset$ & 6 Std. post inj. 3,80 & $\emptyset$ \\
\hline 2. & $17,5 \mathrm{~g}$ subkutan & schwach & schwach + & - & - \\
\hline 3. & - & $\emptyset$ & $+t$ & 24 Std, postinj. 6,50 & 9 \\
\hline
\end{tabular}




\begin{tabular}{|c|c|c|c|c|c|}
\hline \multirow{2}{*}{ Tag } & \multirow{2}{*}{ Urea-Zufuhr } & \multicolumn{2}{|c|}{ Urin } & \multicolumn{2}{|l|}{ Blut } \\
\hline & & Albumen & Indikan & Harnstoff $\%$ & Indikan \\
\hline 4. & $10 \mathrm{~g}$ subkatan & $\not$ & ++ & - & - \\
\hline 5 & $7,5 \mathrm{~g}$ subkutan & $\begin{array}{l}20 \text { Minute } \\
\text { Injektion } \\
\text { mortem }\end{array}$ & $\begin{array}{l}\text { nach der } \\
\text { kurz ante } \\
\text { getötet }\end{array}$ & $\begin{array}{l}20 \text { Minuten post inj. } \\
11,18\end{array}$ & $\emptyset$ \\
\hline
\end{tabular}

2. $2360 \mathrm{~g}$ schweres Kaninchen erhält $40 \mathrm{ccm}$ einer $50 \%$ igen UreaLösung per os. 2 Standen darauf Exitas. Im Urin kein Indikan. Im Blut 10 pro mille Harnstoff, kein Indikan.

Diese nur 2 Stunden ertragene Azotämie genügte also, trotz ihrer abnormen Höhe, wegen der kurzen Dauer der Einwirkung nicht, eine vermehrte Indikanbildung za veranlassen.

3. $2632 \mathrm{~g}$ schweres Kaninchen erhält $10 \mathrm{ccm}$ einer $50 \%$ igen UreaLösung per os, nachdem an 2 Vortagen der Urin frei von Eiweiß und Indikan war. Darauf keine Indikanurie, Blutharnstoff am nächsten Morgen normal $(0,50$ pro mille), kein Blutindikan machweisbar. Am zweiten Tage $20 \mathrm{ccm}$ einer $50 \%$ igen Urea-Lösung per os, darauf im Urin Indikan + , am dritten Tage ++ , am vierten Tage $\varnothing$. Blutharnstoff 20 Stunden nach Eingabe des Harnstoffs 0,55 pro mille.

Nunmehr erhielt das Tier 7 Tage lang täglich $10 \mathrm{ccm}$ der $50 \%$ igen Urea-Lösing, die es gut ausschied, so daß der Blutharnstoff am Morgen nach der Fütterung normal war $(0,20$ pro mille $)$ Es trat keine Indikanurie auf. Am achten und neunten Tage wurden je $15 \mathrm{ccm}$ der Lösnng eingeführt. Auch jetzt entstand keine Indikanurie.

Nach mehreren Tagen erhielt das Tier wieder 2 Tage lang je $15 \mathrm{cem}$ der Lösung per os. Als wieder keine Indikanurie auftrat, wurden am dritten Tage $20 \mathrm{ccm}$ der Lösung gegeben. Nun enthielt der Urin des dritten Tages Indikan schwach + , der des vierten Indikan + (ohne neue Urea-Zufuhr), der des fünften und der folgenden Tage Indikan $\varnothing$.

Eine Woche später erhielt das Tier wieder $20 \mathrm{~cm}$ per os. Keine Indikanurie. 2 Tage später wurden $10 \mathrm{ccm}$, am nächsten Tage $20 \mathrm{ccm}$, am übernächsten Tage wieder $20 \mathrm{ccm}$ per os verabfolgt, ohne daß Indikanurie auftrat. 2 Tage darauf erhielt das Tier $30 \mathrm{ccm}$ mit dem gleichen negativen Erfolg. Weitere 2 Tage später wurden $40 \mathrm{ccm}$ verabfolgt, 45 Minuten darauf Exitus unter Krämpfen.

Warum bei diesem Tier, das anfangs anf die Urea-Zufuhr von $10 \mathrm{~g}$ prompt mit einer Indikanurie reagierte, später auch bei höheren Dosen keine Indikanurie mehr anftrat, können wir nicht erklären. Vielleicht muß man an eine Gewöhnung des Organismus denken. Die Resorption der Urea-Zulage war jedenfalls nicht gestört, da in allen Versuchen mindestens $90 \%$ des mehr zugeführten $\mathrm{N}$ im Urin ausgeschieden wurde.

4. $1650 \mathrm{~g}$ schweres Kaninchen erhält, nachdem der Urin 6 Tage lang frei von Indikan war, $20 \mathrm{ccm}$ einer $50 \%$ igen Urea-Lösung per os. Keine Indikanurie. Am nächsten Tage werden $25 \mathrm{ccm}$ der Lösung gegeben, darauf im Urin Indikan +. 2 Tage später erhält das Tier $30 \mathrm{ccm}$ per os, keine Indikanurie. Am nächsten Tage wieder dieselbe Dosis, ohne 
daß Indikanurie auftritt. Wir sehen hier also ein ähnliches Verhalten wie beim vorigen Tier.

5. $2050 \mathrm{~g}$ schweres Kaninchen erhält, nachdem der Urin 1 Tag lang indikanfrei war, $10 \mathrm{~cm}$ der Urea-Lösung per os. Keine Indikanurie. Am nächsten Tage auch auf $20 \mathrm{ccm}$ keine Indikanurie. Einige Tage später tritt bei Verabfolgung von $25 \mathrm{ccm} 1 \mathrm{Tag}$ lang eine Spur Indikan im Urin auf.

6. $1760 \mathrm{~g}$ schweres Kaninchen erhält, nachdem der Urin 3 Tage lang indikanfrei war, $20 \mathrm{ccm}$ der Harnstofflösung per os. Darauf im Urin Indikan +. Am folgenden Tage wieder $20 \mathrm{~cm}$ per os, im Urin Indikan ++ . Noch am folgenden Tage schwach positive Indikanurie ohne neue Harnstoffzufuhr, dann kein Indikan mehr im Urin.

Einige Tage darauf erhält das Tier $10 \mathrm{ccm}$ : der Lösung per os. Daranf im Urin Spuren Indikan, die am folgenden Tage wieder verschwunden sind.

7. $2660 \mathrm{~g}$ schweres Kaninchen erhält, nachdem der Urin 3 Tage vorher indikanfrei war, $10 \mathrm{ccm}$ der Lösung per os. Keine Indikanurie. Am folgenden Tage $20 \mathrm{cem}$ per os, im Urin Spur Indikan. Am nächsten Tage nach $20 \mathrm{ccm}$ wieder Spur Indikan. Dann keine Indikanurie mehr bei Absetzen der Urea-Zufuhr.

8. Etwa $4000 \mathrm{~g}$ schweres Kaninchen erhält, nachdem der Urin 2 Tage lang indikanfrei war, $30 \mathrm{cem}$ der Lösung per os. Keine Indikanurie. Am nächsten Tage wieder $30 \mathrm{ccm}$ per os. Jetzt Indikanreaktion im Urin +, dann, nach Absetzen der Urea-Zufuhr, 0.

Aus den vorstehenden Versuchen geht folgendes hervor:

Wenn man bei Kaninchen eine vorübergehende künstliche Azotämie ohne Schädigung der Nieren durch übermäBige Harnst offzufuhr erzeugt, tritt in der Mehrzahl der Fälle eine vorher nicht nachweisbare Indikanurie auf. Die Menge des dazu erforderlichen Harnstoffs schwankt etwas, im Allgemeinen sind bei einem etwa $5 \mathrm{Pfd}$. schweren Tier etwa $10 \mathrm{~g}$ Harnstoff erforderlich; es entsteht dabei eine einige Stunden anhaltende Azotämie von ungefähr $3-4$ pro mille Blutharnstoff, die am nächsten Tage wieder abgeklungen ist. Zuweilen tritt die Indikanurie erst am nächsten Tage bei Wiederholung der gleichen Dosis auf, zuweilen läßt sie sich auch dadurch erzielen, daß man am ersten Tage 5, am zweiten Tage $10 \mathrm{~g}$ Harnstoff zuftuhrt; einzelne Tiere reagieren sogar schon auf einmalige Zufuhr von $5 \mathrm{~g}$ Harnstoff mit Indikanurie. Worauf dieses verschiedene Verhalten der einzelnen Tiere beruht, ließ sich bisher nicht ermitteln. Für das. Zustandekommen der Indikanurie scheint jedenfalls ein mehr als zweistiindiges Bestehen der Azotämie erforderlich zu sein, da bei dem zweiten Tier trotz höchster Azotämie, die aber nur 2 Stunden ertragen wurde, keine Indikanurie auftrat. In der geringeren Zahl der Fälle ließ sich trotz 
reichlichster Urea-Zufuhr keine vermehrte Indikanausscheidung bewirken, insbesondere ist auffallend, daß dasselbe Tier anfang's auf die Urea-Zufuhr mit Indikanurie reagierte, später nicht mehr. Man muß hier vielleicht an eine Gewöhnung des Organismus an den erhöhten Harnstoffspiegel denken. A.ls wichtig möchten wir noch hervorheben, daß eine wesentliche Erhöhung des Blutindikans, die sich mit der Obermeyer-T'sehertk of fschen Mikromethode nachweisen ließ, nicht vorhanden war, daß aber geringere Erhöhungen, wie sie sich mit der Jollesschen Probe nachweisen lassen, vorkommen, wie dies bei jeder vermehrten Indikanurie eintreten wird. Wir konnten bei stärkerer Indikanurie noch in 2,5 ccm Serum $(5 \mathrm{ccm}$ Serum-Trichloressigsäurefiltrat) mit der Jollesschen Probe nachweisen.

Es ergibt sich also aus diesen Versuchen ein weiteres Resultat: Wir hatten vorhin gesehen, daß die Azotämie bei der akuten Nephritis von einer gewissen Höhe an mit vermehrter Indikanbildung einhergeht. Nunmehr können wir feststellen, daB diese vermehrte Indikanbildung auch dann in der Mehrzahl der Fälle auftritt, wenn man eine Azotämie ohne Nierenschädigung hervorruft. In beiden Fällen kommt es zunächst zu einer vermehrten Indikanausscheidung im Urin (außer bei Anurie), wobei das Blutindikan nur wenig, entsprechend der Hyperindikanurie, ansteigt. Bei der Azotämie mit Störung der Nierenfunktion kommt nun das Retentionsmoment hinzu, das mehrgebildete Indikan wird bei zunehmender Niereninsuffizienz mehr und mehr im Blut zurückgehalten, und immer weniger gelangt in den Urin. Infolgedessen erreicht die Hyperindikanämie höhere, mit der Obermeyer-Tschertk offschen Methode faßbare Werte. Bei der Azotämie ohne Niereninsuffizienz bleibt die mit dieser Probe nachweisbare Hyperindikanämie aus, das mehrgebildete Indikan passiert nur das Blut von seiner Bildungsstätte bis zu den Nieren und wird hier in den Urin ausgeschieden. Die vermehrte Indikanbildung ist aber beiden Formen der Azotämie gemeinsam und es hat demnach den Anschein, daß die Erhöhung des Blutharnstoffs gewissermaßen aktiv an der vermehrten Indikanbildung beteiligt ist, daß also die vermehrte Indikanbildung und damit auch die Hyperindikanämie eine Folge der Azotämie ist. Die Frage, wo die vermehrte Indikanbildung: statt hat, läßt sich anch in diesem Falle unseres Erachtens nicht mit Sicherheit entscheiden. Man könnte vielleicht daran denken, daß bei der Azotämie mit und ohne Nierenschädigung in der Leber, 
der nach den Untersuchungen von Gautier und Hervieux ${ }^{1}$ ) eine wichtige Rolle bei der Oxydation des Indols zum Indoxyl zukommt, auf den Reiz des azotämischen Blates hin eine pathologische Indikanbildung oder Indikanmehrbildung stattfindet, und daß das mehrgebildete Indikan von hier aus in den Kreislauf gelangt. Zur Beantwortung dieser Frage sind noch weitere Untersuchungen (Durchblutung tiberlebender Leber mit azotämischem Blut) erforderlich. In der Galle solcher künstlich azotämisch gemachter Kaninchen konnten wir jedenfalls kein Indikan nachweisen.

Es lag nun ferner die Frage nahe, ob die Hyperindikanurie infolge kiunstlicher Rest-N-Erhöhung bei nierengesunden Tieren eine spezifische Wirkung des Harnstoffs ist, oder ob sie in gleicher Weise auftritt, wenn man den Rest- $\mathrm{N}$ statt durch Harnstoff durch andere einfach gebaute N-haltige Körper ansteigen läßt. Wir haben auch zur Beantwortung dieser Frage einige Versuche unternommen und einige Tiere mit Ammoniumsalzen (Ammoniumchlorid und Ammoniumzitrat) iuberlastet. Diese Versuche sind bisher daran gescheitert, daß die Tiere die für diesen Zweck erforderliche Dosis nicht vertrugen, sondern kurze Zeit nach der Vergiftung unter Krämpfen eingingen. Wir müssen also diese Frage zunächst noch offen lassen.

Wir haben uns bei den vorstehenden Untersuchungen absichtlich auf die akute Urämie und auf die experimentelle Urämie der Kaninchen beschränkt. Bei der akuten Urämie des Menschen, sei es infolge Vergiftungen ( $\mathrm{Hg}$, Oxalsäure), sei es infolge einer akuten Glomerulonephritis, scheinen die Verhältnisse, soweit wir bis jetzt sehen, aber durchans analog zu liegen. Wie es sich mit der Hyperindikanämie bei chronischen Nephritiden verhält, können wir auf Grund unserer bisherigen Versuche noch nicht entscheiden. Es scheint, als ob hier die Indikanretention eine wesentlichere Rolle für das Zustandekommen der Hyperindikanämie spielt als die Indikanmehrbildung, die aber anscheinend anch statt hat. Der Lösung dieser Frage soll durch weitere Untersuchungen näher getreten werden.

\section{Zusammenfassung.}

1. Es wird an der Hand von experimentellen Nierenschädigungen bei Kaninchen gezeigt, daß der bei der akuten Azotämie auftretenden Hyperindikanämie eine vermehrte Indikanurie vorausgeht, daß es sich also bei dieser Hyperindikanämie zunächst nicht um vermehrte Indikanretention, sondern um ver-

1) Journ. de physiol. et pathol. gen. Bd. 9, S. 593. 
mehrte Indikanbildung handelt. Erst bei weiter fortschreitender Niereninsuffizienz kommt auch eine zunehmende Retention zu der vermehrten Bildung hinzu.

2. Es wird die Frage besprochen, ob diese vermehrte Indikanbildung im Darm oder im intermediären Stoffwechsel vor sich geht. Eine sichere Entscheidung dieser Frage läßt sich zur Zeit nicht treffen, jedoch scheinen die bisher vorliegenden Tatsachen mehr in dem Sinne einer intermediären Indikanbildung zu sprechen. Vielleicht kann die Leber als Ort dieser Mehrbildung angesehen werden.

3. Es wird gezeigt, daß anch die künstliche Azotämie durch Harnstoffzufubr bei nierengesunden Kaninchen meist zu einer Hyperindikanurie, d. h. also zu einer vermehrten Indikanbildung führt. Eine stärkere Hyperindikanämie bleibt hier wegen fehlender Indikanretention aus.

4. Es ist infolgedessen wahrscheinlich, daß die Azotämie in ursächlichem Zusammenhang mit der Hyperindikanämie steht, in dem Sinne, daß die Azotämie die vermehrte Indikanbildung anregt. 